\title{
Integrated microarray analysis provided novel insights to the pathogenesis of glaucoma
}

\author{
JINHUI WANG ${ }^{1 *}$, DAOFEI QU ${ }^{2 *}$, JINGHONG AN $^{1}$, GUOMING YUAN ${ }^{1}$ and YUFU LIU ${ }^{2}$ \\ Departments of ${ }^{1}$ Clinical Laboratory and ${ }^{2}$ Ophthalmology, \\ The First Hospital of Harbin, Harbin, Heilongjiang 150001, P.R. China
}

Received January 23, 2017; Accepted August 15, 2017

DOI: $10.3892 / \mathrm{mmr} .2017 .7711$

\begin{abstract}
Glaucoma is characterized as a visual field defect, which is the second most common cause of blindness. The present study performed an integrated analysis of microarray studies of glaucoma derived from Gene Expression Omnibus (GEO). Following the identification of the differentially expressed genes (DEGs) in glaucoma compared with normal control (NC) tissues, the functional annotation, glaucoma-specific protein-protein interaction (PPI) network and transcriptional regulatory network constructions were performed. The acute intraocular pressure (IOP) elevation rat models were established and reverse transcription-quantitative polymerase chain reaction (RT-qPCR) was performed for DEGs expression confirmation. Three datasets were downloaded from GEO. A total of 97 DEGs, 82 upregulated and 15 downregulated were identified in glaucoma compared with $\mathrm{NC}$ groups with false discovery rate $<0.05$. Response to virus and immune response were two significantly enriched GO terms in glaucoma. Valine, leucine and isoleucine degradation was a significantly enriched pathway of DEGs in glaucoma. According to the PPI network, HDAC1, HBN, UBR4 and PDK1 were hub proteins in glaucoma. FOXD3, HNF-4 and
\end{abstract}

Correspondence to: Professor Yufu Liu, Department of Ophthalmology, The First Hospital of Harbin, 151 Diduan Street, Daoli, Harbin, Heilongjiang 150001, P.R. China

E-mail: liuyufu1504@163.com

${ }^{*}$ Contributed equally

Abbreviations: BMP, bone morphogenetic protein; DEGs, differentially expressed genes; FOXC1, forkhead box $\mathrm{C} 1$; $\mathrm{GEO}$, Gene Expression Omnibus; HEPH, hephaestin; IOP, intraocular pressure; NC, normal control; NMDA, N-Methyl-D-aspartic acid; ONH, optic nerve head; PBX1, PBX homeobox 1; POAG, primary open angle glaucoma; PPI, protein-protein interaction; PWM, Position Weight Matrix; RFTN1, raftlin, lipid raft linker 1; RGCs, retinal ganglion cells; SB, sodium butyrate; SELENBP1, selenium binding protein 1; VPA, valproic acid

Key words: glaucoma, integrated analysis, differentially expressed genes, transcription factor, protein-protein interaction network
AP-1 were the three transcription factors (TFs) derived from top 10 TFs which covered the majority of downstream DEGs in glaucoma. Based on the RT-qPCR results, the expression levels of 3 DEGs, raftlin, lipid raft linker 1 (RFTN1), PBX homeobox 1 (PBX1), HDAC1 were significantly upregulated and the expression of GEM was significantly downregulated in acute IOP elevation rat model at the first and fifth day. These four DEGs had the same expression pattern with our integrated analysis. Therefore, the current study concluded that 6 DEGs, including HEPH, SELENBP1, RFTN1, ID1, HDAC-1 and PBX1 and three TFs, including FOXD3, HNF-4 and AP-1 may be involved with the pathogenesis of glaucoma. The findings of the current study may improve diagnosis and drug design for glaucoma.

\section{Introduction}

As the second most common cause of blindness (1), glaucoma is characterized by a visual field defect induced by progressive loss of retinal ganglion cells (RGCs) and damage to the optic nerve head $(\mathrm{ONH})(1,2)$. Glaucoma may be classified into primary open angle glaucoma (POAG) and closed angle glaucoma according to the angle at the junction between the cornea and iris. POAG is the most common form of glaucoma (3). Glaucoma is a multifactorial disease. Elevated intraocular pressure (IOP) is a major risk factor for glaucoma (4). In addition, old age, family history, racial differences, severe myopia and low diastolic perfusion were also associated with glaucoma (5-8). Various genetic factors, such as vascular endothelial growth factor (VEGF), forkhead box C1 (FOXC1), glutathione S-transferase mu 1 (GSTM1) and cross-linked actin networks (CLANs) has been identified to be associated with glaucoma.

Previous research has shown that lowering IOP is the only clinical treatment demonstrated to slow down the progression of glaucoma (9). Identification of differentially expressed genes (DEGs) in a disease compared with normal control is an approach to find the key genes and pathways associated with the process of diseases and may provide clues for the pathogenesis and identify novel diagnostic and therapeutic strategies. The advance of next generation sequencing technologies allowed for improved identification of DEGs in glaucoma based on previous microarray studies (10-14). Due to the differences in sample size, microarray platforms and analytical techniques 
among the multiple microarray studies, more accurate profiles of DEGs with larger sample size than an individual microarray can be obtained by integrated analysis of multiple microarrays studies (15).

In order to elucidate the cellular and molecular events associated with glaucoma, the present study identified DEGs associated with glaucoma by integrated microarray analysis of glaucoma. Functional annotation and protein-protein interaction (PPI) network construction were performed to identify glaucoma-associated DEGs and pathways to interpret the biological functions of DEGs. Dysregulation of transcription factors (TFs) has been indicated to be involved with the pathogenesis of various diseases such as glaucoma, the glaucoma-specific transcriptional regulatory network of TFs and DEGs was constructed. In addition, the expression of DEGs was confirmed in the acute IOP elevation rat model. The findings of the current study may provide novel insight in the pathogenesis of glaucoma.

\section{Materials and methods}

Eligible gene expression profile of glaucoma. Based on the Gene Expression Omnibus database (GEO; www.ncbi.nlm. nih.gov/geo), the microarray datasets of optic nerve head/optic nerve head astrocytes in glaucoma and normal control (NC) groups were selected. In addition, the selected datasets were the expression profile of whole-genome sequencing and were normalized or original.

Statistical analysis. The present study used the metaMA package in $\mathrm{R}$ version 3.2.3 to combine data from multiple microarray datasets and obtained the individual P-values (16). DEGs in glaucoma compared with NC were identified with False discovery rate (FDR) $<0.05$. The heat-map of DEGs in glaucoma was obtained by using the heatmap. 2 function in the Rgplots package (17).

Functional annotation. Functional annotation of DEGs in glaucoma was performed using the Kyoto Encyclopedia of Genes and Genomes (KEGG) pathway and Gene Ontology (GO) enrichment analyses with the online software GeneCodis3 (genecodis.cnb.csic.es/analysis). FDR $<0.05$ was considered to be statistically significant.

PPI network constriction. PPI network was used to visualize protein-protein interactions. Using Biological General Repository for Interaction Datasets (BioGRID) (thebiogrid. org/) and Cytoscape version 3.3.0 (www.cytoscape.org/), the PPI network of the top 20 upregulated DEGs and downregulated DEGs in glaucoma were constructed. Nodes were used to represent the proteins and edges were used to represent the interaction between two proteins.

Construction of glaucoma-specific transcriptional regulatory network. The promoter regions of top 20 up and downregulated DEGs in glaucoma were obtained using the University of California Santa Cruz (UCSC) Genome Browser (genome. ucsc.edu). The online tool TRANFAC (18) was used to obtain TFs and motif sequences of binding sites and position weight matrix (PWM). The present study used PWM-scan to obtained the TFs which regulate proteins encoded by DEGs and constructed glaucoma-specific transcriptional regulatory network of top 20 upregulated and downregulated DEGs using Cytoscape version 3.3.0.

Acute elevation of IOP. A total of 9 male Sprague-Dawley rats (age, 8-10 weeks; weight, $300 \mathrm{~g}$ ) were housed under normal atmosphere with controlled-temperature $\left(25^{\circ} \mathrm{C}\right)$, illumination (12-h light/dark cycle) and with free access to food and water. The 9 rats were divided randomly into control group, model 1 day group and model 5 day group. Except control group, the acute IOP elevation rat model was established by saline perfusion into anterior chamber as previously described (19). IOP was raised by elevating the reservoir of saline $120 \mathrm{~cm}$ above the animal's eye for $1 \mathrm{~h}$. IOP was measured using an Icare TonoLab tonometer (ICare Finland Oy, Vantaa, Finland).

$R T-q P C R$ validation of DEGs. After the acute IOP elevation rat model establishment, the rat eyeball tissues in control group $(n=3)$, model 1 day group $(n=3)$ and model 5 day group $(n=3)$ were obtained prior to acute IOP elevation rats model establishment and on the first and fifth day after acute IOP elevation rats model establishment. The total RNA was extracted with TRIzol reagent (Invitrogen; Thermo Fisher Scientific, Inc., Waltham, MA, USA). cDNA was generated from extracted RNA with SuperScript III Reverse Transcriptase (Invitrogen; Thermo Fisher Scientific, Inc.) incubated at $42^{\circ} \mathrm{C}$ for $1 \mathrm{~h}$ and $72^{\circ} \mathrm{C}$ for $10 \mathrm{~min}$. In an ABI 7500 real-time PCR system (Applied Biosystems; Thermo Fisher Scientific, Inc.), qPCR was performed with Power SYBR-Green PCR Master mix (Thermo Fisher Scientific, Inc.). Cycling conditions were $95^{\circ} \mathrm{C}$ for $15 \mathrm{~min}$ followed by 40 cycles of $95^{\circ} \mathrm{C}$ for $10 \mathrm{sec}, 55^{\circ} \mathrm{C}$ for $30 \mathrm{sec}$ and $72^{\circ} \mathrm{C} 32 \mathrm{sec}$. The relative gene expression of selected DEGs was analyzed using $2^{-\Delta \Delta C q}$ method (20). The forward and reverse primers (Majorbio Co., Shanghai, China) are presented in Table I. The human 18srRNA was used as endogenous control for mRNA expression in analysis. Quantification of mRNA expression was performed using GraphPad Prism version 5 (GraphPad Inc., La Jolla, CA).

\section{Results}

Identification of DEGs in glaucoma. Three datasets, including GSE45570, GSE9944 and GSE2378 were used in the current study (Table II). A total of 97 DEGs (82 upregulated and 15 downregulated were identified in glaucoma samples compared with the NC group with FDR $<0.05$. The heatmap of DEGs in glaucoma was illustrated in Fig. 1 and the top 20 DEGs in glaucoma were displayed in Table III.

Functional annotation. According to the GO enrichment analysis, response to virus $\left(\mathrm{FDR}=7.11 \times 10^{-5}\right)$, immune response $(\mathrm{FDR}=0.00252621)$, protein binding $\left(\mathrm{FDR}=2.59 \times 10^{-10}\right)$, transcription factor binding $(\mathrm{FDR}=0.000806942)$, nucleus $\left(\mathrm{FDR}=1.24 \times 10^{-6}\right)$, extracellular space $(\mathrm{FDR}=0.000618804)$ were significantly enriched GO terms of DEGs in glaucoma. The top 20 significantly enriched GO terms including biological process, cellular component and molecular function of DEGs in glaucoma were displayed in Fig. 2. After the KEGG enrichment analysis, Valine, leucine and isoleucine 
Table I. Primers used in reverse transcription quantitative polymerase chain reaction.

Gene $\quad$ Primers $\left(5^{\prime}-3^{\prime}\right)$

RFTN1

BCKDHB

CASP1

NMI

PLPP3

DCLK1

KCTD12

HEPH

ID1

ABCA8

MID1

PBX1

SEPP1

FUT8

NBN

SELENBP1

HDAC1

AMIGO2

GEM

CYFIP2
Forward: agaaggcagagcttcccaat Reverse: aggtgttcttccatcctg Forward: atactttaccgggcagcagt Reverse: ttttcttgggccatggaagc Forward: acaaagaaggtggcgcattt Reverse: aacatcagctccgactctcc Forward: atatcgatggggacgcatgt Reverse: cctcagacagttcatcggga Forward: ccttatgtggecgctctcta Reverse: tggcccgagaagaaggattt Forward: ccatcgtgacatcaagccag Reverse: aggccatatccagtctctgc Forward: atggaggagagggagcagta Reverse: caaaacgacaccaggacag Forward: accttagctggcaccttgat Reverse: tctgtccgtggaagaaagct Forward: aacagcaggtgaacgttctg Reverse: tcgcgacttcagactcagag Forward: aaacagcccaccaactccta Reverse: ttcatcctgggttgtctgct Forward: ttgtgtaaactggttgggcg Reverse: cttggcttcttgacgggatg Forward: agccgaattgcaggtctttc Reverse: tctcctcttccagcctttg Forward: ggtttgcectactcettcet Reverse: ttgtcatggtgcttgtggtg Forward: gaaaattcacttcggggcgt Reverse: tctggttgtgggcattttgg Forward: cagagctggcagttcaagtg Reverse: tcctcactgctgtcctgaag Forward: atacatgtgtgggactggca Reverse: gtagaagcgctggatgttgg Forward: tacgacggggatgttggaaa Reverse: ttggtcatctcctcagcgtt Forward: tgccatgttccaggagctaa Reverse: agatcagccagcttgaacct Forward: tctgcggtggaagagttgat Reverse: tctttgggtcaatctgggct Forward: acttcctcccaactactgc Reverse: gcggtaggagctgtagatgt

RFTN1, raftlin, lipid raft linker 1; PBX1, PBX homeobox 1; ID1, inhibitor of differentiation 1; SELENBP1, selenium binding protein 1.

degradation $(\mathrm{FDR}=0.0213357)$, Cytokine-cytokine receptor interaction $(\mathrm{FDR}=0.0220177)$, Glycolysis/gluconeogenesis (FDR=0.0293935) were most significantly enriched pathways of DEGs in glaucoma. The significantly enriched KEGG pathways of DEGs in glaucoma were displayed in Fig. 3 and Table IV.
Glaucoma-specific PPI network. A PPI network (Fig. 4) of the top 20 upregulated DEGs in glaucoma was constructed, which consisted of 813 nodes and 865 edges. The hub proteins were HDAC1 (degree=456), HBN (degree=75) and MID1 (degree=61). The PPI network (Fig. 5) of the downregulated DEGs in glaucoma was constructed, which consisted of 372 nodes and 393 edges. The hub proteins were UBR4 (degree=56), PDK1 (degree=45) and PHKG2 (degree=41).

Glaucoma-specific transcriptional regulatory network. The glaucoma-specific transcriptional regulatory network of top 20 upregulated DEGs (Fig. 6) was consisted of 57 nodes and 141 edges. The glaucoma-specific transcriptional regulatory network of downregulated DEGs (Fig. 7) consisted of 53 nodes and 103 edges. The top 10 TFs covering the majority of downstream DEGs in glaucoma were presented in Table V.

Acute elevation of IOP and RT-qPCR validation of DEGs. IOP was significantly increased in model 1 day group and model 5 day group after the acute IOP elevation rats model establishment. In order to verify the expression of integrated analysis, the top 20 DEGs in glaucoma were investigated. Based on the RT-qPCR (Fig. 8), the expression of 3 DEGs [raftlin, lipid raft linkerm 1 (RFTN1), PBX homeobox 1 (PBX1), HDAC1] were significantly upregulated and the expression of GEM was significantly downregulated in the acute IOP elevation rat model on the first and fifth day. These four DEGs presented the same pattern in the integrated analysis in the present study.

\section{Discussion}

In order to reveal the cellular and molecular events associated the pathogenesis of glaucoma, the present study identified several genes and TFs that were involved with glaucoma based on the DEGs in glaucoma compared with the NC group by using an integrated microarray analysis.

The acute IOP elevation rat model was established to validate the expression of DEGs in glaucoma using RT-qPCR. The acute IOP elevation model has been previously used to simulate acute angle-closure glaucoma $(21,22)$; however, the association between acute IOP elevation and chronic glaucoma remains to be fully elucidated. Acute ischemic insult and non-ischemic injuries may be induced by the acute IOP elevation model and lead to significant loss of RGCs after 3 days of acute IOP elevation (23). Alterations in the morphology of astrocytes and influence on axons and cellular responses within the ONH may also be induced by acute IOP elevation $(24,25)$. Additionally, Morrison et al previously reported that gene expression profiles within the $\mathrm{ONH}$ induced by acute IOP elevation were similar to those observed following chronic IOP elevation $(26,27)$. Due to the similarity between the acute IOP elevation model and glaucoma, the gene expression profiles in acute IOP model may identify the key genes in glaucoma.

A previous study has indicated that the $\mathrm{ONH}$ is repeatedly exposed to cycles of relatively minor injuries in the early stage of glaucoma (28) and these minor injuries may be reversed by treatment with healthy RGCs (28). However, repeated insults or an intrinsic impairment may overwhelm the capacity of RGCs to recover, and the cell death program may be induced (29). 


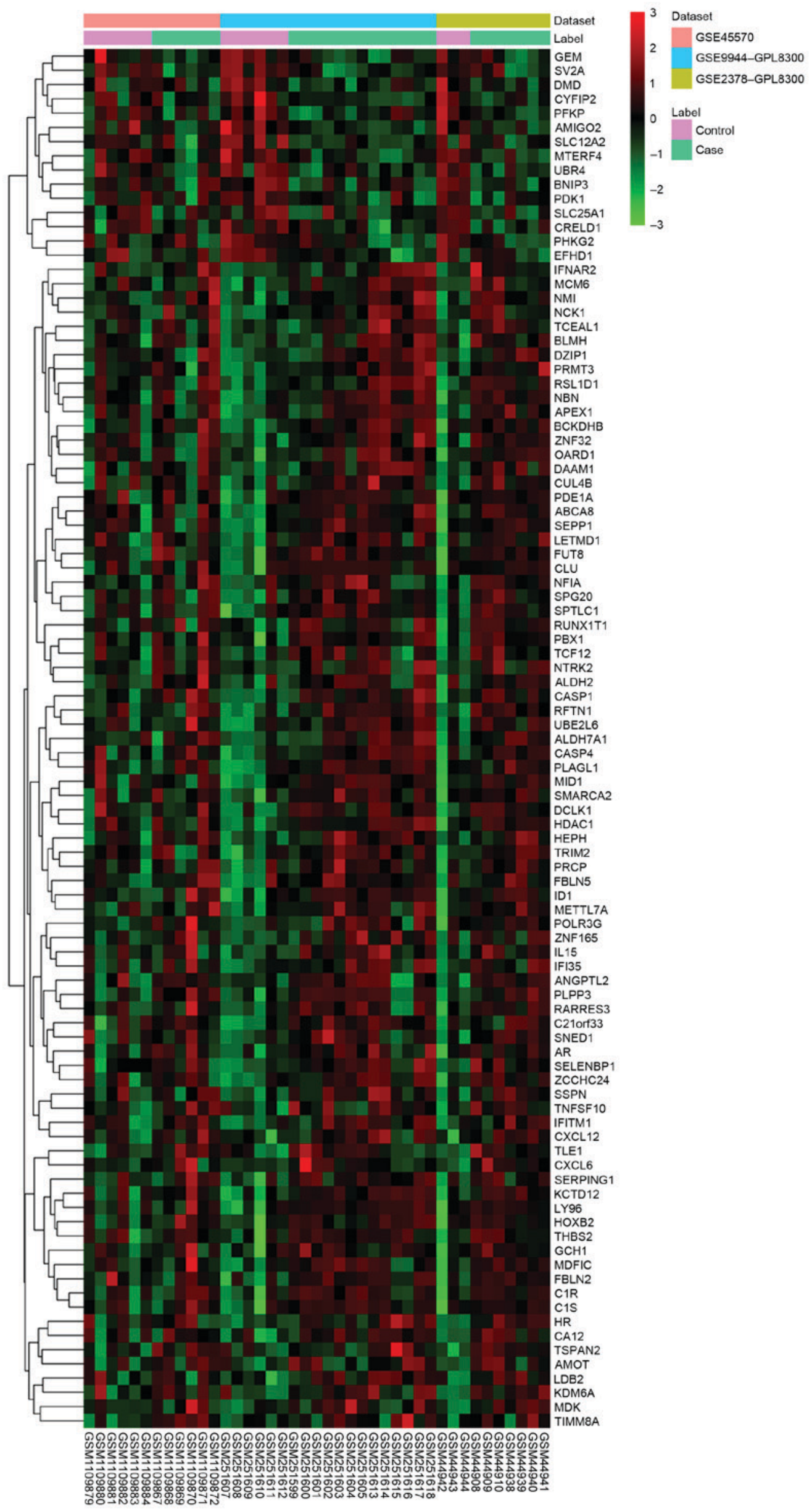

Figure 1. Heatmap of differentially expressed genes in glaucoma samples compared with normal control. 


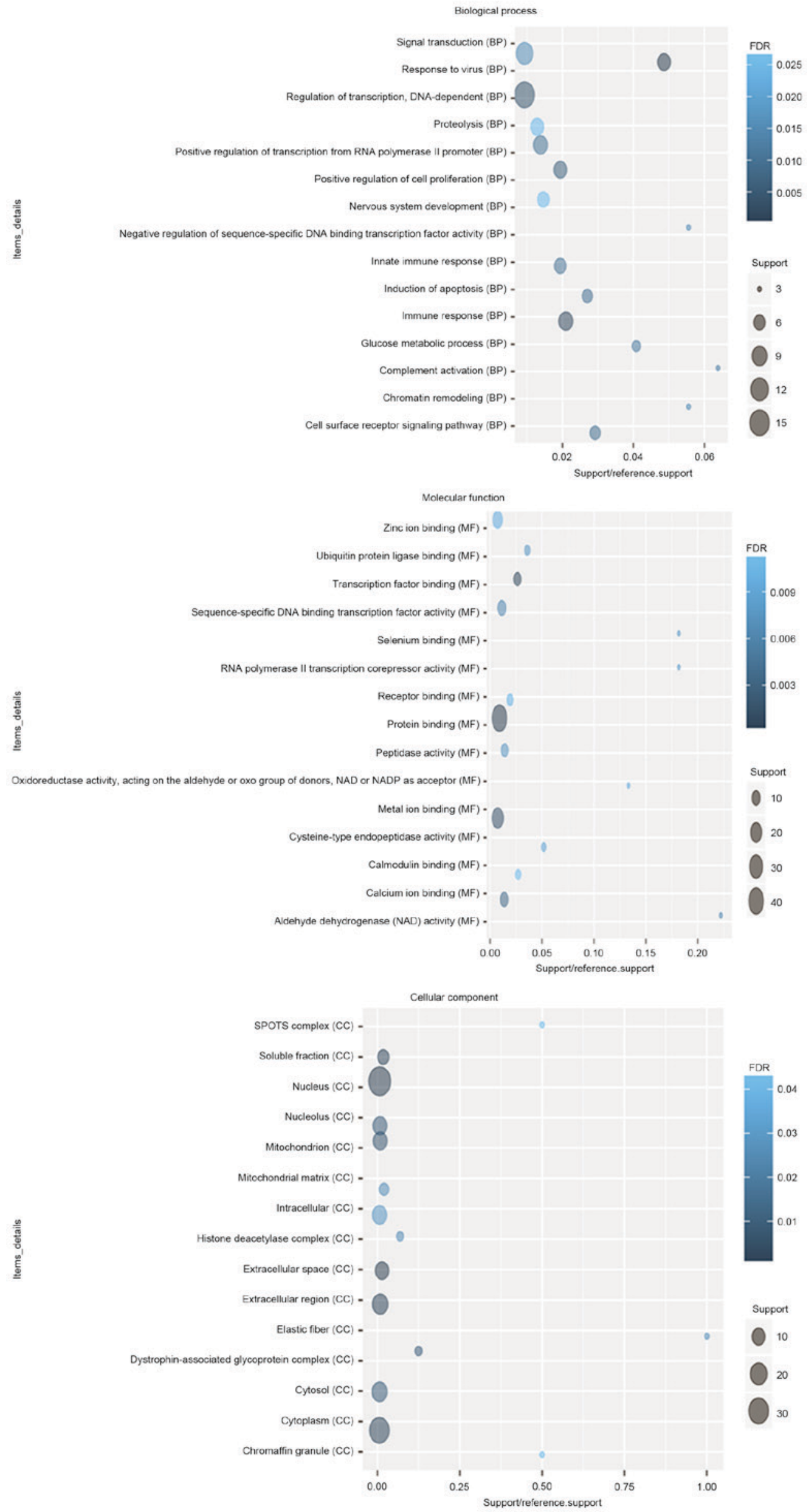

Figure 2. Top 20 significantly enriched Gene Ontology terms of differentially expressed genes in glaucoma compared to normal control. 
Table II. mRNA expression datasets used in this study.

\begin{tabular}{|c|c|c|c|c|}
\hline GEO accession no. & Platform & Normal:Case & Year & Tissue \\
\hline GSE45570 & GPL5175 & $6: 6$ & 2013 & Optic nerve head \\
\hline GSE9944 & GPL8300 & $6: 13$ & 2008 & Optic nerve head astrocytes \\
\hline GSE2378 & GPL8300 & $3: 7$ & 2005 & Optic nerve head astrocytes \\
\hline
\end{tabular}

Table III. Top 20 differentially expressed genes in glaucoma compared with normal control.

\begin{tabular}{|c|c|c|c|c|c|}
\hline ID & Gene name & Combined ES & P-value & FDR & Regulation \\
\hline 23180 & RFTN1 & 1.97057 & $1.18 \times 10^{-6}$ & 0.001965 & Up \\
\hline 594 & BCKDHB & 1.863234 & $1.38 \times 10^{-6}$ & 0.001965 & Up \\
\hline 834 & CASP1 & 1.901172 & $4.48 \times 10^{-7}$ & 0.001965 & $\mathrm{Up}$ \\
\hline 9111 & NMI & 1.813946 & $1.10 \times 10^{-6}$ & 0.001965 & $\mathrm{Up}$ \\
\hline 347902 & AMIGO2 & -1.86914 & $2.38 \times 10^{-6}$ & 0.002707 & Down \\
\hline 8613 & PLPP3 & 1.69169 & $3.85 \times 10^{-6}$ & 0.002979 & $\mathrm{Up}$ \\
\hline 9201 & DCLK1 & 1.84913 & $4.72 \times 10^{-6}$ & 0.002979 & Up \\
\hline 115207 & KCTD12 & 1.796888 & $5.38 \times 10^{-6}$ & 0.003057 & $\mathrm{Up}$ \\
\hline 2669 & GEM & -1.66006 & $6.53 \times 10^{-6}$ & 0.003149 & Down \\
\hline 9843 & HEPH & 1.663202 & $7.21 \times 10^{-6}$ & 0.003149 & $\mathrm{Up}$ \\
\hline 3397 & ID1 & 1.677848 & $8.45 \times 10^{-6}$ & 0.003428 & Up \\
\hline 10351 & ABCA8 & 1.753583 & $1.68 \times 10^{-5}$ & 0.006363 & Up \\
\hline 4281 & MID1 & 1.770553 & $1.81 \times 10^{-5}$ & 0.006411 & $\mathrm{Up}$ \\
\hline 5087 & PBX1 & 1.616593 & $2.08 \times 10^{-5}$ & 0.006948 & $\mathrm{Up}$ \\
\hline 6414 & SEPP1 & 1.711474 & $2.27 \times 10^{-5}$ & 0.007171 & $\mathrm{Up}$ \\
\hline 2530 & FUT8 & 1.5493 & $2.47 \times 10^{-5}$ & 0.007393 & $\mathrm{Up}$ \\
\hline 3065 & HDAC1 & 1.642544 & $3.09 \times 10^{-5}$ & 0.008781 & $\mathrm{Up}$ \\
\hline 26999 & CYFIP2 & -1.5543 & $4.16 \times 10^{-5}$ & 0.010273 & Down \\
\hline 4683 & NBN & 1.51878 & $3.96 \times 10^{-5}$ & 0.010273 & $\mathrm{Up}$ \\
\hline 8991 & SELENBP1 & 1.577832 & $3.99 \times 10^{-5}$ & 0.010273 & $\mathrm{Up}$ \\
\hline
\end{tabular}

RFTN1, raftlin, lipid raft linker 1; FDR, false discovery rate; ES, effect size; PBX1, PBX homeobox 1; SELENBP1, selenium binding protein 1.

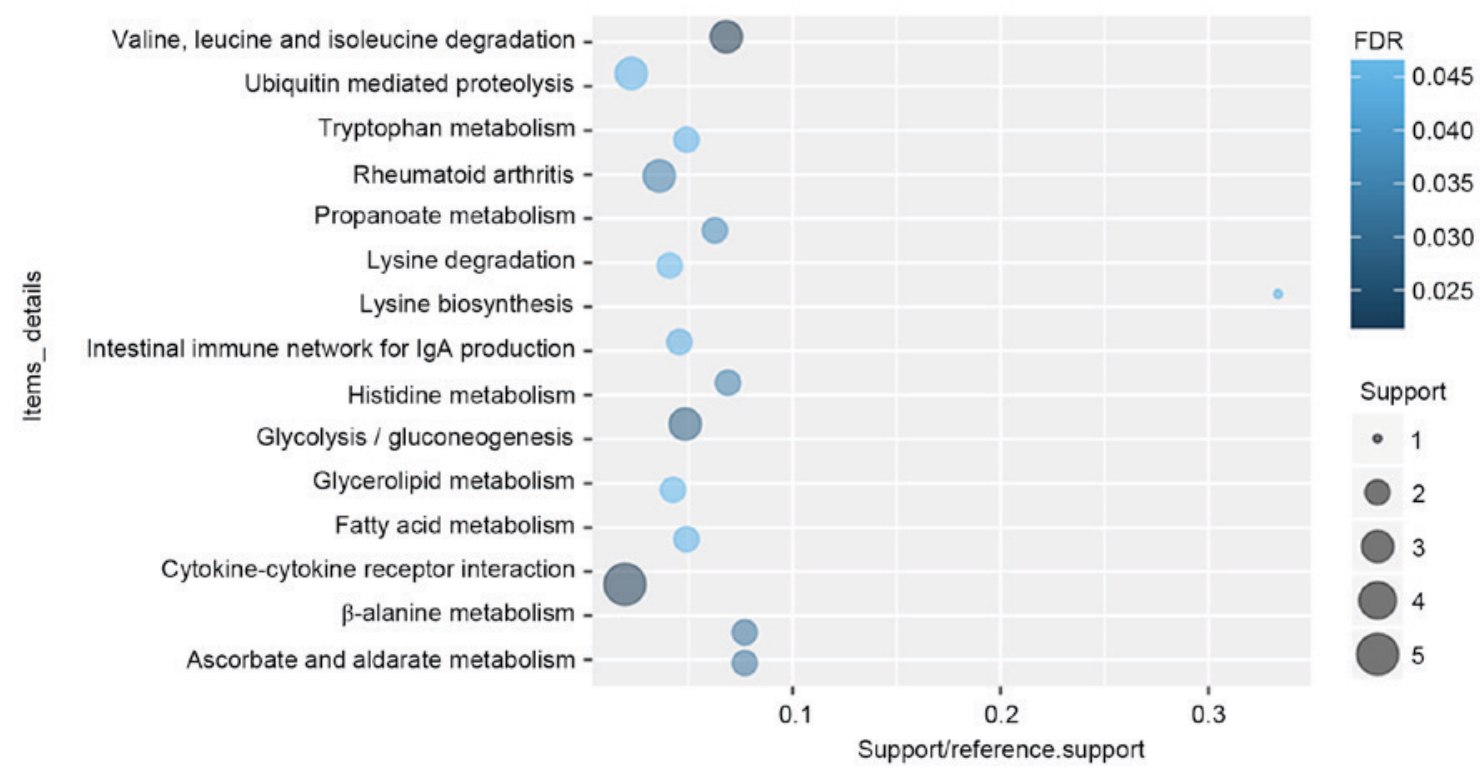

Figure 3. Top 20 significantly enriched Kyoto Encyclopedia of Genes and Genomes pathways of differentially expressed genes in glaucoma compared to normal control. BP, biological processes; MF, molecular function, CC, cellular components; FDR, false discovery rate. 


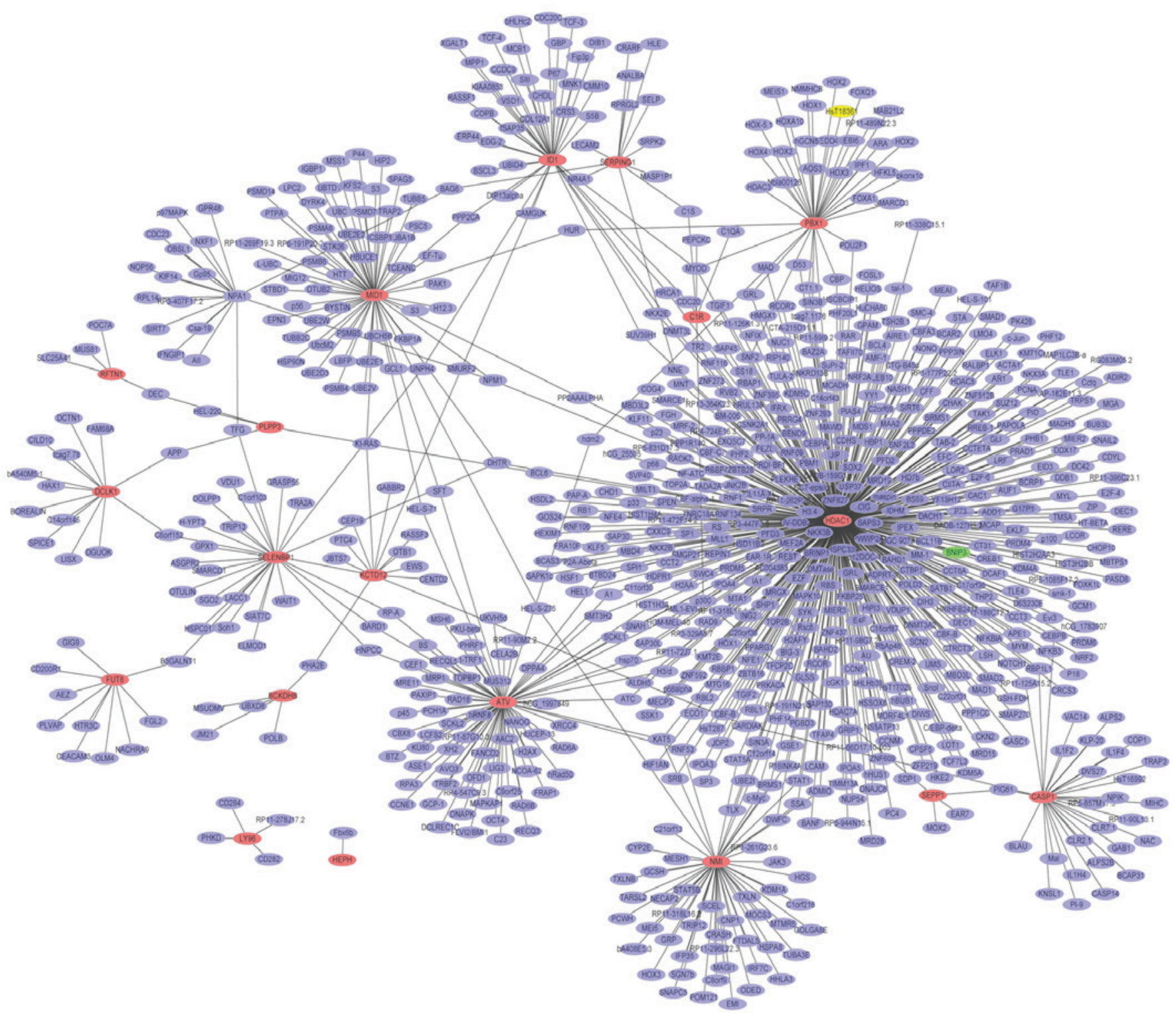

Figure 4. Glaucoma-specific protein-protein interaction network of top 20 upregulated DEGs. The red ellipses indicate the proteins encoded by upregulated DEGs and the purple ellipses indicate other proteins. DEGs, differentially expressed genes.

Although 1 day of acute IOP elevation is insufficient to induce evident damage on the $\mathrm{ONH}$, the present study determined that the process of RGC recovery is activated after 1 day of acute IOP elevation which may be associated with early-stage glaucoma. Therefore, the expression of selected DEGs after 1 day and 5 days was validated.

As the major risk factor of glaucoma, elevated IOP may induce the optic nerve axonal compression at the lamina cribrosa, block the axoplasmic flow, cause interference in retrograde neurotrophin transport to RGCs, and drive apoptosis. This may lead to disruption of the balance between aqueous humor production and outflow from the anterior chamber (30). Elements regulating IOP and aqueous humor may be involved with the pathogenesis of glaucoma.

As a transporter expressed by ciliary body epithelia, RFTN1 was believed to contribute to the pathogenesis of POAG by changing the composition of aqueous humor (31). In addition, previous studies have reported that raftlin, RFTN1 rs690037 was associated with three glaucoma-associated optic disc parameters including optic cup area, vertical cup-to-disc ratio and central corneal thickness $(32,33)$. Combination of RFTN1 and other optic disc parameter-associated genes, such as ATOH7 single nucleotide polymorphisms conferred risk for POAG (32). In the integrated analysis of the present study, RFTN1 was the top upregulated DEG in glaucoma compared with $\mathrm{NC}$ and it was also upregulated in the acute IOP elevation rat model, which provided evidence for the potential role of RFTN1 in glaucoma. In order to determine the specific role of RFTN1 in glaucoma, further research is required.

Hephaestin (HEPH) and selenium binding protein 1 (SELENBP1) were previously reported to be associated with elevated intraocular pressure (34) and upregulated HEPH has been previously detected in glaucoma (35). In the current study, HEPH and SELENBP1 were also upregulated DEGs in glaucoma and the acute IOP elevation rat model. Therefore, it is possible that HEPH and SELENBP1 may be involved with glaucoma by regulating IOP. 
Table IV. Significantly enriched Kyoto Encyclopedia of Genes and Genomes pathways of differentially expressed genes in glaucoma compared with normal control.

\begin{tabular}{|c|c|c|c|c|c|}
\hline ID & Function & Count & P-value & FDR & Genes \\
\hline 00280 & Valine, leucine and isoleucine degradation & 3 & 0.0002452 & 0.0213357 & ALDH7A1, BCKDHB, ALDH2 \\
\hline 04060 & Cytokine-cytokine receptor interaction & 5 & 0.0007592 & 0.0220177 & $\begin{array}{l}\text { CXCL6, IL15, IFNAR2, } \\
\text { CXCL12, TNFSF10 }\end{array}$ \\
\hline 00010 & Glycolysis/gluconeogenesis & 3 & 0.0006757 & 0.0293935 & ALDH7A1, PFKP, ALDH2 \\
\hline 00410 & $\beta$-alanine metabolism & 2 & 0.0023259 & 0.0337254 & ALDH7A1, ALDH2 \\
\hline 00053 & Ascorbate and aldarate metabolism & 2 & 0.0023259 & 0.0337254 & ALDH7A1, ALDH2 \\
\hline 05323 & Rheumatoid arthritis & 3 & 0.0016298 & 0.0354476 & CXCL6, IL15, CXCL12 \\
\hline 00340 & Histidine metabolism & 2 & 0.0028901 & 0.0359192 & ALDH7A1, ALDH2 \\
\hline 00640 & Propanoate metabolism & 2 & 0.0035119 & 0.0381914 & ALDH7A1, ALDH2 \\
\hline 04672 & $\begin{array}{l}\text { Intestinal immune network for } \\
\text { IgA production }\end{array}$ & 2 & 0.0065563 & 0.043877 & IL15, CXCL12 \\
\hline 00300 & Lysine biosynthesis & 1 & 0.0082213 & 0.0447033 & ALDH7A1 \\
\hline 04120 & Ubiquitin mediated proteolysis & 3 & 0.0062022 & 0.0449656 & CUL4B, UBE2L6, MID1 \\
\hline 00071 & Fatty acid metabolism & 2 & 0.0057135 & 0.0451886 & ALDH7A1, ALDH2 \\
\hline 00380 & Tryptophan metabolism & 2 & 0.0057135 & 0.0451886 & ALDH7A1, ALDH2 \\
\hline 00561 & Glycerolipid metabolism & 2 & 0.0074521 & 0.0463094 & ALDH7A1, ALDH2 \\
\hline 00310 & Lysine degradation & 2 & 0.0080782 & 0.0468536 & ALDH7A1, ALDH2 \\
\hline 00330 & Arginine and proline metabolism & 2 & 0.0097429 & 0.0470908 & ALDH7A1, ALDH2 \\
\hline 05130 & Pathogenic Escherichia coli infection & 2 & 0.0097429 & 0.0470908 & NCK1, LY96 \\
\hline
\end{tabular}

FDR, false discovery rate.

However, lowering IOP is not always an effective treatment for patients with glaucoma, which suggested that other mechanisms of glaucoma require further research.

Glaucoma is the most common optic neuropathy and RGCs apoptosis is a major hallmark of glaucoma (36). The present study identified several DEGs in glaucoma which were associated with RGCs damage and apoptosis.

Retinal injury, including light damage and N-MethylD-aspartic acid (NMDA) treatment have been reported to induce an increase in bone morphogenetic protein (BMP) expression and BMP-Smad1/5/8 signaling in inner retinal cells, which suggested that BMP-Smad1/5/8 signaling had a neuroprotective effect on RGCs after damage (37). As a target of BMP-Smad1/5/8, inhibitor of differentiation 1 (ID1) was upregulated in response to retinal injury (37). According to the present study, ID1 was also upregulated in the acute IOP elevation rat model and glaucoma which is a RGCs disease. The current study concluded that ID1 was involved with RGC damage, which may be a potential target for neuroprotective therapies in glaucoma and other RGC diseases.

Nuclear atrophy is one of the early events of RGCs death and previous studies have reported that Class I histone deacetylases (HDACs) 1, 2 and 3 have key roles in nuclear atrophy and apoptosis of RGCs (36). In addition, inhibition of HDACs activity in the RGCs nucleus may prevent RGCs death. Treatment of purified rat RGCs with inhibitors including valproic acid (VPA) and sodium butyrate (SB), prevented histone deacetylation and protected RGCs from death in vitro (38). MS-275, an inhibitor of HDAC1/HDAC3 was identified to have a protective effect on the loss of RGCs and promoted RGC survival following optic nerve injury (39). According to the PPI network of the top 20 upregulated DEGs in glaucoma, HDAC1 is a hub protein which was upregulated in the acute IOP elevation rat model as well. The study concluded that inhibitors of HDACs, particularly the HDAC1 inhibitor may have a protective role of RGCs in glaucoma (39). Further investigation is required to identify the specific roles of each individual HDAC in injured RGCs and determine which HDAC may be the appropriate target for therapeutic inhibition in optic neuropathies such as glaucoma.

According to the GO and KEGG enrichment analysis, various changes occurred in glaucoma compared with $\mathrm{NC}$ and the majority of enriched pathways in glaucoma were metabolic-associated pathways. Glycolysis/gluconeogenesis and valine, leucine and isoleucine degradation were two significantly enriched pathways in glaucoma which were consistent with previous studies (40-42) and may have important roles in glaucoma. These metabolic-associated pathways may involve with the process of glaucoma and these abnormal metabolites may make a contribution in early diagnosis and treatment of glaucoma.

The present study used the glaucoma-specific transcriptional regulatory network, and identified three TFs which may be involved with the process of glaucoma. Ocular malformation is another risk factor of glaucoma, which may be induced by dysplasia of the anterior eye (43). The structures of anterior eye are closely associated with the migratory neural crest cells. The FOXD3 encodes a forkhead 


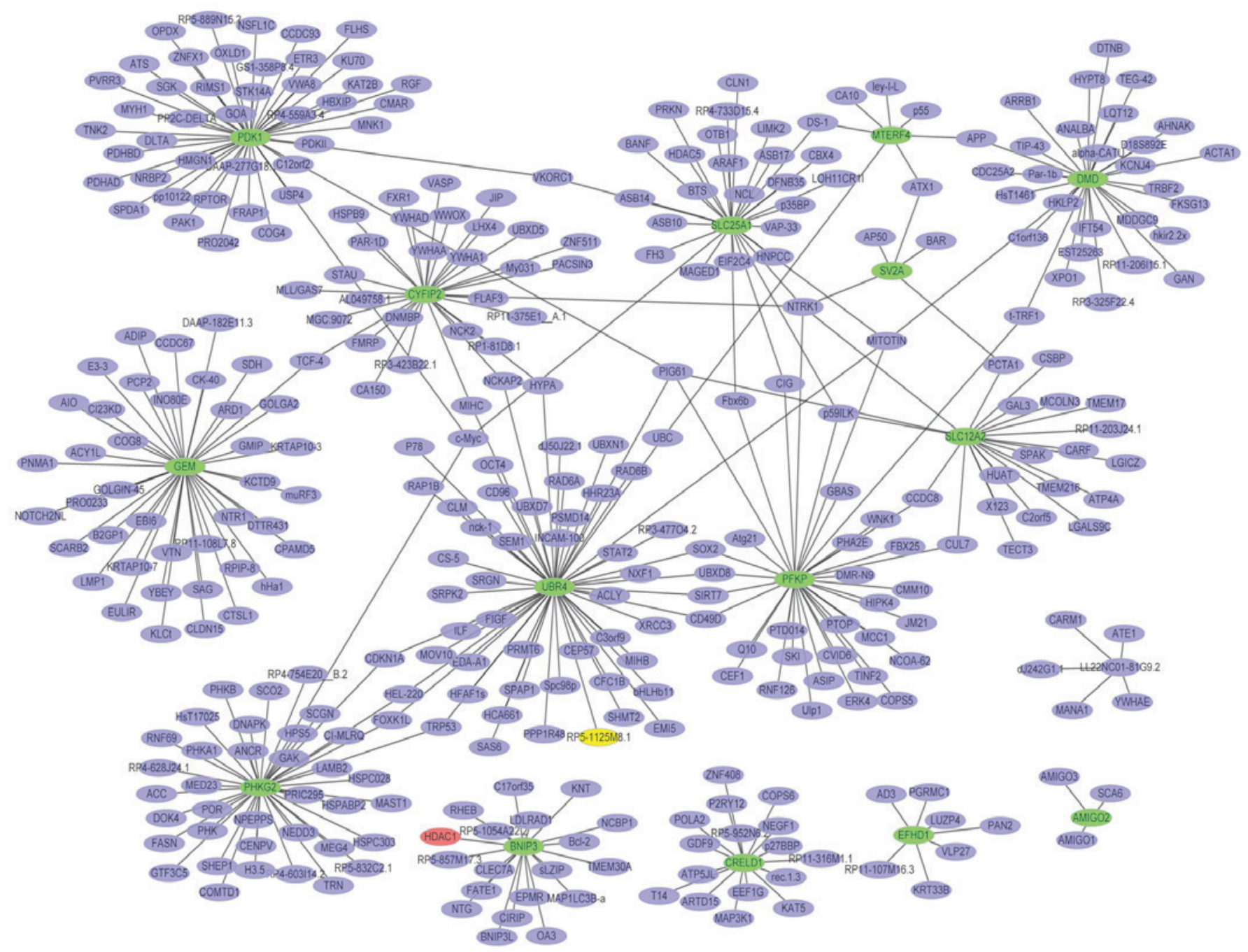

Figure 5. Glaucoma-specific protein-protein interaction network of top 20 downregulated DEGs. The green ellipses indicate the proteins encoded by downregulated DEGs and the purple ellipses indicate the other proteins. DEGs, differentially expressed genes.

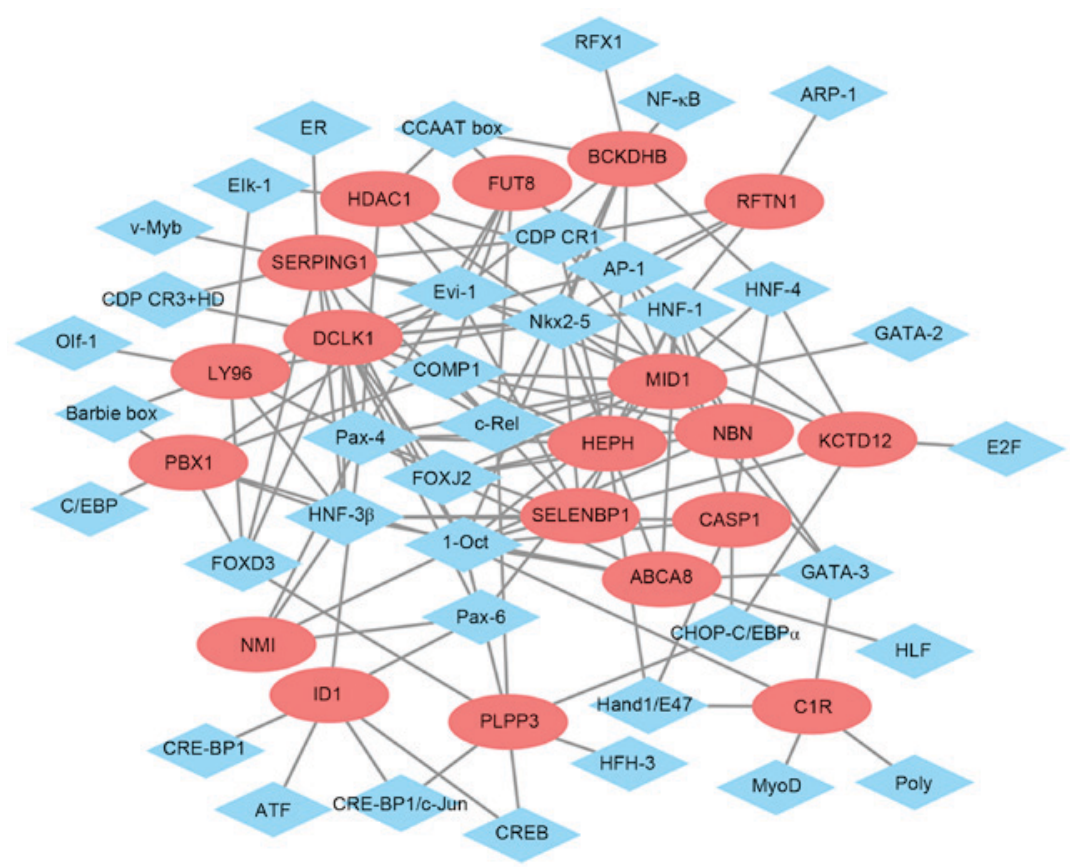

Figure 6. Transcriptional regulatory network of top 20 upregulated DEGs in glaucoma. The red ellipses indicate the upregulated DEGs and the blue rhombus indicate the TFs. The lines represent the TF-DEG pairs. DEGs, differentially expressed genes; TF, transcription factor. 
Table V. Top 10 TFs covering the majority of downstream DEGs in glaucoma.

\begin{tabular}{|c|c|c|}
\hline TF & Count & DEGs \\
\hline Pax-4 & 50 & $\begin{array}{l}\text { SERPING1, SV2A, EFHD1, NMI, DCLK1, CRELD1, CRELD1, AMIGO2, NBN, SERPING1, NBN, } \\
\text { NBN, SLC12A2, NBN, FUT8, SELENBP1, SERPING1, SLC25A1, EFHD1, FUT8, LY96, EFHD1, } \\
\text { HEPH, ID1, SERPING1, CYFIP2, UBR4, ABCA8, HDAC1, GEM, ID1, SELENBP1, PHKG2, } \\
\text { SERPING1, EFHD1, CRELD1, CRELD1, ID1, LY96, SERPING1, ID1, LY96, HDAC1, EFHD1, } \\
\text { SELENBP1, LY96, DMD, MID1, HDAC1 }\end{array}$ \\
\hline $1-\mathrm{Oct}$ & 44 & $\begin{array}{l}\text { DMD, DMD, MID1, DMD, NBN, DMD, MID1, PDK1, BCKDHB, BCKDHB, NBN, DMD, } \\
\text { BCKDHB, DMD, MID1, DMD, KCTD12, PLPP3, NMI, C1R, DMD, PBX1, SERPING1, DMD, } \\
\text { UBR4, SERPING1, MID1, DCLK1, KCTD12, ABCA8, PDK1, SERPING1, DCLK1, PBX1, } \\
\text { KCTD12, DMD, PHKG2, PBX1, NBN, BCKDHB, MID1, CASP1, NBN, DMD }\end{array}$ \\
\hline Nkx2-5 & 38 & $\begin{array}{l}\text { HEPH, NBN, SV2A, NBN, NBN, SERPING1, NBN, SERPING1, ABCA8, EFHD1, DMD, HEPH, } \\
\text { SELENBP1, LY96, DCLK1, SELENBP1, PHKG2, SV2A, HDAC1, SLC12A2, BCKDHB, EFHD1, } \\
\text { DMD, BNIP3, EFHD1, SERPING1, AMIGO2, RFTN1, SERPING1, ABCA8, ABCA8, SELENBP1, } \\
\text { UBR4, EFHD1, LY96, KCTD12, ABCA8, KCTD12 }\end{array}$ \\
\hline COMP1 & 26 & $\begin{array}{l}\text { SLC12A2, MID1, MID1, SLC25A1, MID1, UBR4, DMD, PBX1, HEPH, FUT8, DMD, MID1, NBN, DMD, } \\
\text { NBN, MID1, NBN, DCLK1, NBN, PHKG2, DMD, MID1, PBX1, SELENBP1, SLC25A1, DCLK1 }\end{array}$ \\
\hline FOXD3 & 23 & $\begin{array}{l}\text { DMD, DMD, SLC25A1, PBX1, PLPP3, SERPING1, PLPP3, DMD, DCLK1, DCLK1, SERPING1, } \\
\text { PLPP3, DMD, PDK1, DMD, DMD, SERPING1, LY96, SLC25A1, CYFIP2, UBR4, DMD, PDK1 }\end{array}$ \\
\hline HNF-1 & 23 & $\begin{array}{l}\text { ABCA8, DMD, DMD, DMD, RFTN1, PHKG2, HEPH, DMD, SV2A, DMD, CYFIP2, DMD, } \\
\text { AMIGO2, MID1, SELENBP1, FUT8, CASP1, NBN, NBN, KCTD12, DCLK1, DCLK1, DMD }\end{array}$ \\
\hline HNF-4 & 20 & $\begin{array}{l}\text { MID1, GEM, PDK1, BNIP3, BCKDHB, CYFIP2, SLC25A1, UBR4, MID1, SLC25A1, BNIP3, } \\
\text { DMD, MID1, SLC25A1, KCTD12, CYFIP2, SLC12A2, PDK1, SLC25A1, CASP1 }\end{array}$ \\
\hline Evi-1 & 18 & $\begin{array}{l}\text { HDAC1, DMD, MID1, BCKDHB, FUT8, EFHD1, LY96, DMD, DMD, BCKDHB, DMD, MID1, } \\
\text { PBX1, PBX1, SERPING1, SELENBP1, PHKG2, FUT8 }\end{array}$ \\
\hline AP-1 & 15 & $\begin{array}{l}\text { NBN, HEPH, HEPH, PHKG2, MID1, HDAC1, RFTN1, PHKG2, BCKDHB, DCLK1, CYFIP2, } \\
\text { DCLK1, FUT8, PHKG2, BNIP3 }\end{array}$ \\
\hline $\mathrm{HNF}-3 \beta$ & 12 & PBX1, SERPING1, LY96, NMI, SELENBP1, DMD, SERPING1, DCLK1, ABCA8, DMD, CASP1, DMD \\
\hline
\end{tabular}

TFs, transcription factor; DEGs, differentially expressed genes; PBX1, PBX homeobox 1; RFTN1, raftlin, lipid raft linker 1; ID1, inhibitor of differentiation 1 .

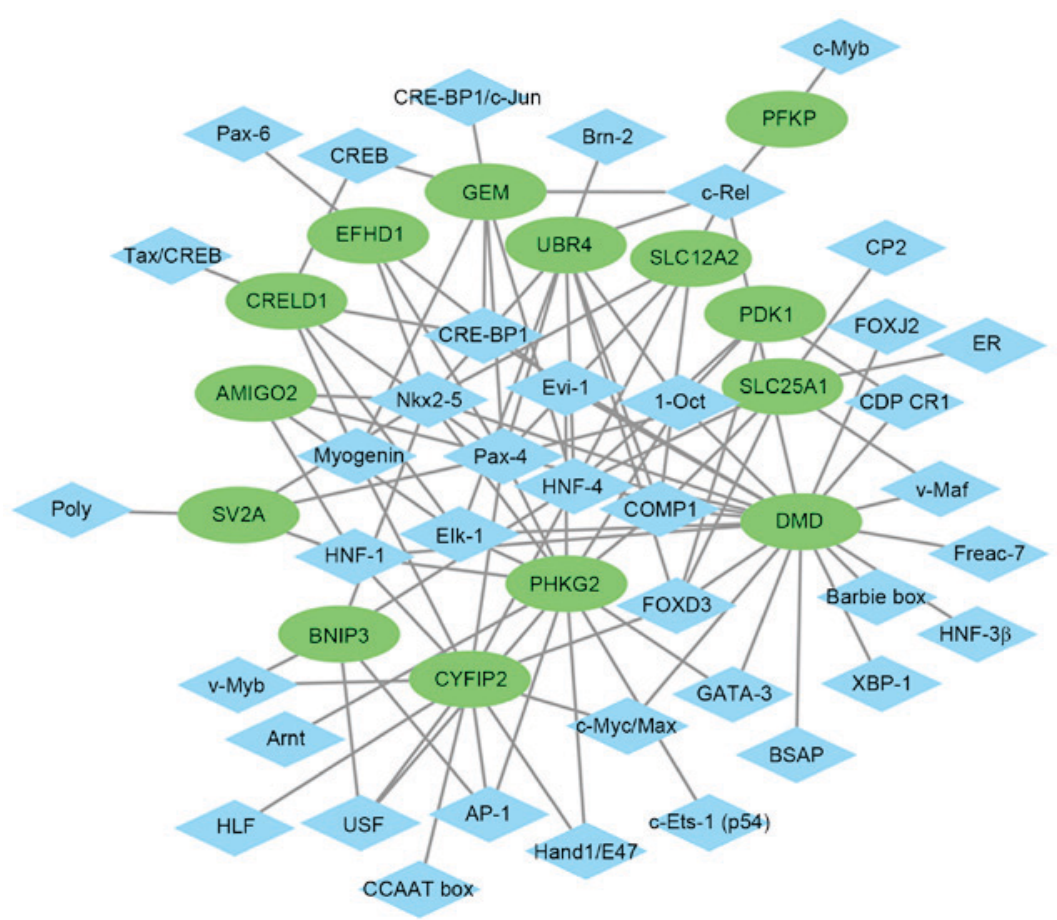

Figure 7. Transcriptional regulatory network of downregulated DEGs in glaucoma. The green ellipses represented the downregulated DEGs and the blue rhombus indicated the TFs. The lines represent the TF-DEG pairs. DEGs, differentially expressed genes; TF, transcription factor. 


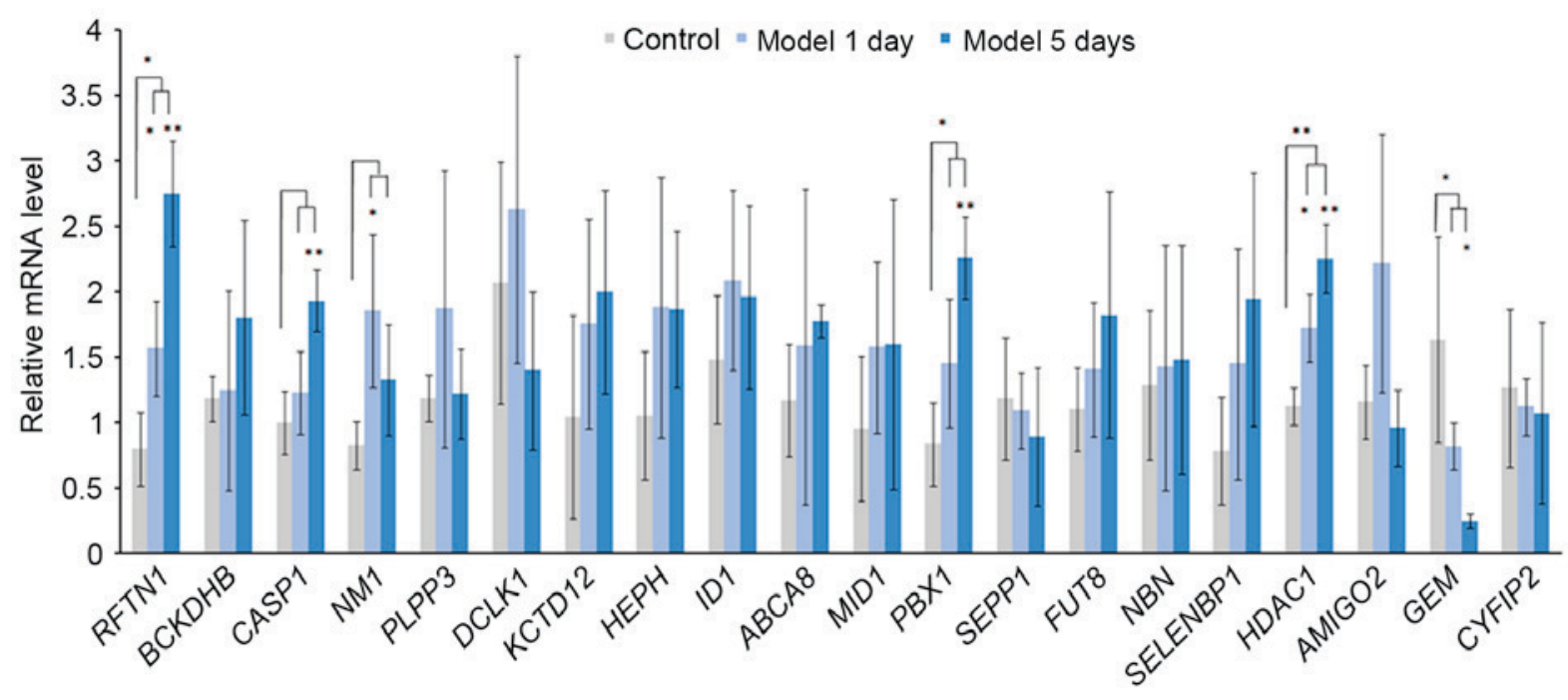

Figure 8. Reverse transcription-quantitative polymerase chain reaction results of the top 20 differentially expressed genes in acute intraocular pressure elevation rat models. ${ }^{*} \mathrm{P}<0.05$ and ${ }^{* *} \mathrm{P}<0.01$ vs. control group.

TF, which has a crucial role in neural crest specification in vertebrates; therefore, FOXD3 may be involved in multiple types of eye diseases, such as glaucoma (44). Previous studies have indicated that FOXC1 has a role in various glaucoma phenotypes $(45,46)$. Additionally, another upregulated DEG in glaucoma and the acute IOP elevation rat model, PBX1 may impair the activity of FOXC1 in a filamin A-mediated manner. Considering the present findings and previous studies, it is possible that PBX1 may be involved with the process of glaucoma by interacting with FOXC1. HNF-4 and AP-1 were two TFs identified from top 10 TFs covering the majority of downstream DEGs which have a role in cellular regulation of the reactive $\mathrm{ONH}$ astrocytes (11). Therefore, the present study concluded that HNF-4 and AP-1 may be closely associated with glaucoma.

Additionally, to the best of our knowledge this is the first study to report the association between glaucoma and other 6 DEGs, including NMI, KCTD12, ABCA8, MID1, FUTB, and GEM, which were also confirmed by RT-qPCR in the acute IOP elevation rat model; however, their potential roles in glaucoma require further investigation.

In conclusion, HEPH, SELENBP1 and RFTN1 may be involved with the pathogenesis of glaucoma by regulating IOP or glaucoma-associated optic disc parameters. ID1 and HADC1 may be associated with glaucoma by the influence on RGCs. Interaction between PBX1 and FOXC1 may have a role in glaucoma. Three TFs, including FOXD3, HNF-4 and AP-1 may also act as regulators of glaucoma. The findings of the present study may contribute to developing novel and more effective approaches of diagnosis and drug design for glaucoma. Future investigations of glaucoma-associated genes, require the chronic IOP elevation model and the specific role of these glaucoma-associated DEGs and TFs in glaucoma necessary in further research.

\section{Acknowledgements}

The present study was funded by Talent introduction subsidy program of Harbin (grant no. 2014SYYRCYJ06-2).

\section{References}

1. Yu-Wai-Man P, Stewart JD, Hudson G, Andrews RM, Griffiths PG, Birch MK and Chinnery PF: OPA1 increases the risk of normal but not high tension glaucoma. J Med Genet 47: 120-125, 2010.

2. Moreno MC, Sande P, Marcos HA, de Zavalía N, Keller Sarmiento MI and Rosenstein RE: Effect of glaucoma on the retinal glutamate/glutamine cycle activity. FASEB J 19: 1161-1162, 2005.

3. Liu T, Xie L, Ye J and He X: Family-based analysis identified $\mathrm{CD} 2$ as a susceptibility gene for primary open angle glaucoma in Chinese Han population. J Cell Mol Med 18: 600-609, 2014.

4. Guo MS, Wu YY and Liang ZB: Hyaluronic acid increases MMP-2 and MMP-9 expressions in cultured trabecular meshwork cells from patients with primary open-angle glaucoma. Mol Vis 18: 1175-1181, 2012.

5. Gazzard G, Foster PJ, Devereux JG, Oen F, Chew P, Khaw PT and Seah S: Intraocular pressure and visual field loss in primary angle closure and primary open angle glaucomas. Br J Ophthalmol 87: 720-725, 2003.

6. Rosenberg LF: Glaucoma: Early detection and therapy for prevention of vision loss. Am Fam Physician 52: 2289-2298, 1995.

7. Bikbova G, Oshitari T and Yamamoto S: Diabetes mellitus and retinal vein occlusion as risk factors for open angle glaucoma and neuroprotective therapies for retinal ganglion cell neuropathy. J Clin Exper Ophthalmol S3, 2012.

8. McMenemy MG: Primary Open Angle Glaucoma. Springer, New York, NY, pp1721-1721, 2014.

9. Girard MJA, Zimmo L, White ET, Mari JM, Ethier CR and Strouthidis NG: Towards a biomechanically-based diagnosis for glaucoma: In vivo deformation mapping of the human optic nerve head. In: ASME 2012 Summer Bioengineering Conference. Puerto Rico, pp423-424, 2012.

10. Wu Y, Zang WD and Jiang W: Functional analysis of differentially expressed genes associated with glaucoma from DNA microarray data. Genet Mol Res 13: 9421-9428, 2014.

11. Lukas TJ, Miao H, Chen L, Riordan SM, Li W, Crabb AM, Wise A, Du P, Lin SM and Hernandez MR: Susceptibility to glaucoma: Differential comparison of the astrocyte transcriptome from glaucomatous African American and Caucasian American donors. Genome Biol 9: R111, 2008.

12. Hernandez MR, Agapova OA, Yang P, Salvador-Silva M, Ricard CS and Aoi S: Differential gene expression in astrocytes from human normal and glaucomatous optic nerve head analyzed by cDNA microarray. Glia 38: 45-64, 2002.

13. Yan X, Yuan F, Chen X and Dong C: Bioinformatics analysis to identify the differentially expressed genes of glaucoma. Mol Med Rep 12: 4829-4836, 2015.

14. Rozsa FW, Scott KM, Pawar H, Samples JR, Wirtz MK and Richards JE: Differential expression profile prioritization of positional candidate glaucoma genes: The GLC1C locus. Arch Ophthalmol 125: 117-127, 2007. 
15. Fei Q, Lin J, Meng H, Wang B, Yang Y, Wang Q, Su N, Li J and Li D: Identification of upstream regulators for synovial expression signature genes in osteoarthritis. Joint Bone Spine 83: 545-551, 2016

16. Marot G, Foulley JL, Mayer CD and Jaffrézic F: Moderated effect size and P-value combinations for microarray meta-analyses. Bioinformatics 25: 2692-2696, 2009.

17. Wei K, Pan S and Li Y: Functional characterization of maize $\mathrm{C}_{2} \mathrm{H}_{2}$, zinc-finger gene family. Plant Mol Biol Rep 34: 761-776, 2016 .

18. Matys V, Fricke E, Geffers R, Gössling E, Haubrock M, Hehl R, Hornischer K, Karas D, Kel AE, Kel-Margoulis OV, et al: TRANSFAC: Transcriptional regulation, from patterns to profiles. Nucleic Acids Res 31: 374-378, 2003.

19. Adachi M, Takahashi K, Nishikawa M, Miki H and Uyama M: High intraocular pressure-induced ischemia and reperfusion injury in the optic nerve and retina in rats. Graefes Arch Clin Exp Ophthalmol 234: 445-451, 1996.

20. Livak KJ and Schmittgen TD: Analysis of relative gene expression data using real-time quantitative PCR and the 2(-Delta Delta C(T)) method. Methods 25: 402-408, 2001.

21. Adachi M, Takahashi K, Nishikawa M, Miki H and Uyama M: High intraocular pressure-induced ischemia and reperfusion injury in the optic nerve and retina in rats. Graefes Arch Clin Exp Ophthalmol 234: 445-451, 1996.

22. Huang Y, Li Z, Wang N, van Rooijen N and Cui Q: Roles of $\mathrm{PI} 3 \mathrm{~K}$ and JAK pathways in viability of retinal ganglion cells after acute elevation of intraocular pressure in rats with different autoimmune backgrounds. BMC Neurosci 9: 78, 2008.

23. Zhang S, Wang H, Lu Q, Qing G, Wang N, Wang Y, Li S, Yang D and Yan F: Detection of early neuron degeneration and accompanying glial responses in the visual pathway in a rat model of acute intraocular hypertension. Brain Res 1303: 131-143, 2009.

24. Sun D, Qu J and Jakobs TC: Reversible reactivity by optic nerve astrocytes. Glia 61: 1218-1235, 2013.

25. Lye-Barthel M, Sun D and Jakobs TC: Morphology of astrocytes in a glaucomatous optic nerve. Invest Ophthalmol Vis Sci 54: 909-917, 2013.

26. Morrison JC, Cepurna WO, Doser TA, Dyck JA and Johnson EC: A short interval of controlled elevation of iop (CEI) reproduces early chronic glaucoma model optic nerve head $(\mathrm{ONH})$ gene expression responses. Invest Ophthalmol Vis Sci 51: 5216, 2010.

27. Morrison JC, Cepurna WO, Tehrani S, Choe TE, Jayaram H, Lozano DC, Fortune B and Johnson EC: A period of controlled elevation of IOP (CEI) produces the specific gene expression responses and focal injury pattern of experimental rat glaucoma. Invest Ophthalmol Vis Sci 57: 6700-6711, 2016.

28. Downs JC,Burgoyne CF, Seigfreid WP, Reynaud JF, Strouthidis NG and Sallee V: 24-hour IOP telemetry in the nonhuman primate: Implant system performance and initial characterization of IOP at multiple timescales. Invest Ophthalmol Vis Sci 52: 7365-7375, 2011.

29. Crowston JG, Kong YX, Trounce IA, Dang TM, Fahy ET, Bui BV, Morrison JC and Chrysostomou V: An acute intraocular pressure challenge to assess retinal ganglion cell injury and recovery in the mouse. Exp Eye Res 141: 3-8, 2015

30. Ashworth Briggs EL, Toh T, Eri R, Hewitt AW and Cook AL: TIMP1, TIMP2 and TIMP4 are increased in aqueous humor from primary open angle glaucoma patients. Mol Vis 21: 1162-1172, 2015.

31. Janssen SF, Gorgels TG, van der Spek PJ, Jansonius NM and Bergen AA: In silico analysis of the molecular machinery underlying aqueous humor production: Potential implications for glaucoma. J Clin Bioinforma 3: 21, 2013.
32. Chen JH, Wang D, Huang C, Zheng Y, Chen H, Pang CP and Zhang M: Interactive effects of ATOH7 and RFTN1 in association with adult-onset primary open-angle glaucoma. Invest Ophthalmol Vis Sci 53: 779-785, 2012.

33. Macgregor S, Hewitt AW, Hysi PG, Ruddle JB, Medland SE, Henders AK, Gordon SD, Andrew T, McEvoy B, Sanfilippo PG, et al: Genome-wide association identifies ATOH7 as a major gene determining human optic disc size. Hum Mol Genet 19: 2716-2724, 2010

34. Xue W, Du P, Lin S, Dudley VJ, Hernandez MR and Sarthy VP: Gene expression changes in retinal Müller (glial) cells exposed to elevated pressure. Curr Eye Res 36: 754-767, 2011.

35. Futterweit W, Ritch R, Teekhasaenee C and Nelson ES: Coexistence of Prader-Willi syndrome, congenital ectropion uveae with glaucoma, and factor XI deficiency. JAMA 255: 3280-3282, 1986.

36. Schmitt HM, Schlamp CL and Nickells RW: Role of HDACs in optic nerve damage-induced nuclear atrophy of retinal ganglion cells. Neurosci Lett 625: 11-15, 2016.

37. Ueki Y and Reh TA: Activation of BMP-Smad1/5/8 signaling promotes survival of retinal ganglion cells after damage in vivo. PLoS One 7: e38690, 2012.

38. Biermann J, Boyle J, Pielen A and Lagrèze WA: Histone deacetylase inhibitors sodium butyrate and valproic acid delay spontaneous cell death in purified rat retinal ganglion cells. Mol Vis 17: 395-403, 2011

39. Chindasub P, Lindsey JD, Duong-Polk K, Leung CK and Weinreb RN: Inhibition of histone deacetylases 1 and 3 protects injured retinal ganglion cells. Invest Ophthalmol Vis Sci 54: 96-102, 2013.

40. Messina-Baas OM, González-Huerta LM, Chima-Galán C, Kofman-Alfaro SH, Rivera-Vega MR, Babayán-Mena I and Cuevas-Covarrubias SA: Molecular analysis of the CYP1B1 gene: Identification of novel truncating mutations in patients with primary congenital glaucoma. Ophthalmic Res 39: 17-23, 2007.

41. Mansergh FC, Kenna PF, Ayuso C, Kiang AS, Humphries P and Farrar GJ: Novel mutations in the TIGR gene in early and late onset open angle glaucoma. Hum Mutat 11: 244-251, 1998.

42. Nakamura M: New insights into the pathogenesis of glaucomatous optic neuropathy and refinement of the objective assessment of its functional damage. Nippon Ganka Gakkai Zasshi 116: 298-344, 2012.

43. Sowden JC: Molecular and developmental mechanisms of anterior segment dysgenesis. Eye 21: 1310-1318, 2007.

44. Kloss BA, Reis LM, Brémond-Gignac D, Glaser T and Semina EV: Analysis of FOXD3 sequence variation in human ocular disease. Mol Vis 18: 1740-1749, 2012.

45. Lehmann OJ, Ebenezer ND, Jordan T, Fox M, Ocaka L, Payne A, Leroy BP, Clark BJ, Hitchings RA, Povey S, et al: Chromosomal duplication involving the forkhead transcription factor gene FOXC1 causes iris hypoplasia and glaucoma. Am J Hum Genet 67: 1129-1135, 2000.

46. Chakrabarti S, Kaur K, Rao KN, Mandal AK, Kaur I, Parikh RS and Thomas R: The transcription factor gene FOXC1 exhibits a limited role in primary congenital glaucoma. Invest Ophthalmol Vis Sci 50: 75-83, 2009 